\title{
XXXVIII. Die Structur der optisch drehenden Krystalle.
}

\author{
Von \\ L. Sohncke in München. \\ (Hierzu Fig. 1-4 auf Taf. VII und 13 Textfiguren.)
}

1.

Die Drehung der Polarisationsebene in gewissen Krystallen ist von Fresn el bekanntlich darauf zurückgefuhrt worden, dass ein einfallender geradlinig polarisirter Lichtstrahl sich in einen rechts und einen links circularen Strahl zerlege, die mit ungleichen Geschwindigkeiten fortschreiten. Ohne Antwort gelassen hat er aber die tiefor eindringende Frage : Wodurch gerade gewisse Krystalle befähigt werden, die Drehung zu erzeugen? Seine Untersuchung lässt also die eigentliche Ursache dieser merkwürdigen Erscheinung unerk]ärt. Eine befriedigende Beantwortung dieser Frage erhält man erst durch genaueres Eingehen auf die Structu r der optisch drehenden Krystalle.

Nach der von mir entwickelten Theorie der Krystallstructur*) sind die Bausteine eines Krystalles so angeordnet, dass ihre Schwerpunkte entweder ein regelmässiges unendliches Punktsystem oder (in selteneren Fällen) zwei oder mehr ineinander stehende solche Systeme bilden. Diese Theorie stellt für jede der 32 möglichen Krystallklassen eine Anzahl von Structurformen zur Verfügung, welche die erforderlichen Symmetrieeigenschaften besitzen. Somit finden nicht nur alle Voll- und Theilfächner einschliesslich der hemimorphen Gestalten unter jenen Structurformen ihre Repräsentanten, sondern es wird auch die mannigfaltig verschiedene Ausbildungsweise inner-

*) Entwickelung einer Theorie der Krystallstructur. Leipzig 1879. (System Nr. 13 ist zu streichen.) Dann: Erweiterung der Theorie der Krystallstructur. Diese Zeitschr. 1888, 14, 426 .

Groth, Zeitschrift f. Krystallogr. XIX. 
halb desselben Krystallsystems ohne Weiteres begreiflich. Ebenso wirft die Theorie Licht auf viele andere Eigenschaften der Krystalle, wie z. B. auf die Spaltbarkeit, auf die verschiedene Häufigkeit der an einem Krystall möglichen verschiedenen Flächen*); u. s. w.

Darauf nun, dass auch das optische Drehvermögen von Krystallen durch diese Theorie seine unmittelbare Erklärung findet, babe ich schon vor langer Zeit aufmerksam gemacht**), als ich die von Reusch entdeckte optische Drehwirkung gewisser wendeltreppenartig aufgebauter Glimmercombinationen experimentell eingehender untersuchte und theoretisch aus der Undulationstheorie ableitete***). Doch habe ich mich bisher immer mit dem Nachweise begnïgt, dass gewisse Punktsysteme, welche Structuren des rhomboëdrischen Krystallsystems darstellen, in völlig analoger Weise wie jene Glimmercombination aufgebaut sind, so dass dadurch zunächst nur die Structur rhomboëdrischer drehender Substanzen, wie z. B. des Quarzes, als ermittelt gelten konntef). Bezüglich der in andere Krystallsysteme gehörigen drehenden Substanzen wies ich nur darauf hin, dass auch unter den Punktsystemen, welche jenen Krystallsystemen zuzuordnen sind, sich mannigfaltige "Schraubensysteme " finden, obne dass ich jedoch bisher genauer untersucht hätte, ob sich in diesen anderen Fällen das Drehvermögen wirklich in entsprechender Weise wie beim Quarz auf die Structur zurüickführen lässt.

Es ist die Aufgabe des Folgenden, diese Lucke auszufullen. Ich werdezeigen, dass die Theorie in jedem Krystallsysteme, innerhalb dessen man optisch drehende Krystalle kennt, thatsächlich solche Punktsysteme zur Verfügung stellt, deren Bau Drehung der Polarisationsebene zur Folge haben muss. Hierbei wird es nicht nothwendig sein, die complicirteren Systeme, welche aus mehreren ineinander gestellten regelmässigen unendlichen Punktsystemen bestehen, heranzuziehen, weil sich tberall schon einfache regelmässige Punktsysteme von der erforderlichen Beschaffenheit finden. Zugleich wird eine wesentliche Unvollkommenheit beseitigt werden, diemeiner bisherigen Zurückführung der Drehungdes Quarzes auf dieihmzukommende Structur innewo h n te.

*) Ueber Spaltungsflächen u. natürl. Krystallflächen. Diese Zeitschr. 1887, 13, 214.

**) Zur Theorie des optischen Drehvermögens der Krystalle. Mathem. Annalen 1876, 9, 504. Dann: Die Glimmercombination von Reusch und das optische Drehvermögen von Krystallen. In Poggendorff's Annal. 1878, Ergänz.-Bd. 8, 16. Auch »Entwickelung einer Theorie u. s. f. ", S. 238.

***) Die Theorie der Glimmercombinationen ist spater auch von Herrn Mallard und zwar in ungleich erschöpfenderer und allgemeinerer Weise gegeben worden.

t) Vergl. "Ueber Spaltungsflächen u. s. f. “ Diese Zeitschr. 1887, 13, 229. 
Wegen des Folgenden ist es nöthig, kurz an die grundlegenden Be-

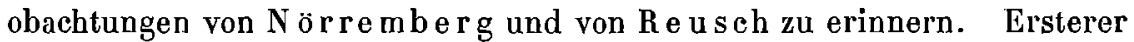
schichtete eine Anzahl Glimmerblättchen von gleicher, aber sehr geringer Dicke, welche derselben Platte zweiaxigen Glimmers entnommen waren, so aufeinander, dass die optische Axenebene eines jeden gegen die des vorhergehenden unter einem rechten Winkel gekreuzt war. Ein solches Präparat verhält sich im Polarisationsapparate fast vollständig wie eine senkrecht zur optischen Axe geschnittene Platte eines optisch einaxigen Krystalls: es zeigt die concentrischen Farbenringe mit dem schwarzen, resp. weissen Kreuze. Reu sch änderte diesen Versuch dahin ab, dass er die aufeinander folgenden Blättchen nicht unter $90^{\circ}$, sondern unter $60^{\circ}$ oder unter $45^{0}$ gegeneinander drehte, und zwar bei demselben Präparate immer in demselben Drehungssinne. Zwischen die einzelnen Glimmerblättchen wird je etwas Copalfirniss gebracht. Eine solche Glimmercombination verhält sich im Polarisationsapparate fast genau wie eine senkrecht zur optischen Axe geschnittene Platte eines optisch einaxigen, die Polarisationsebene drehenden Krystalls. Diese optische Drehung erfolgt rechts herum, d. h. im Uhrzeigersinne, wenn jedes weiter aufgelegte Blättchen en tge $\mathrm{g}$ e $\mathrm{n}$ dem Uhrzeigersinne um $60^{\circ}$ (oder um 450) gegen das vorhergehende gedreht ist. Bei Aufschichtung mit entgegengesetztem Drehungssinne wird auch die optische Drehung die entgegengesetzte.

Unter den mit dreizähliger Hauptaxe begabten regelmässigen Punktsystemen, welche also Structuren des $r h$ o m boëd $\mathrm{r}$ is chen Krystallsystems darstellen, finden sich nun solche, welche folgende Auffassung zulassen. Eine Schaar von Punkten ist auf zwei zur Hauptaxe senkrechten Nachbarebenen angeordnet; sie besitzt - für sich betrachtet - den geometrischen Charakter eines monoklinen Krystalls, entspricht also völlig dem einzelnen Glimmerblättchen*). Nun gehört eine gewisse Schraubung um die dreizählige Hauptaxe zu den charakteristischen Deckbewegungen jener Systeme

*) Wenn Herr F ock (Zur Erklarung der opt. Activität. Ber. d. deutschen chem. Ges. 1894, Jahrg. 24, Heft 1, S. 102) meint, dass ich einer einzelnen parallel zur Basis gelegenen Netzebene die Eigenschaft einer zweiaxigen Lamelle zuschreibe, so hat er meine früheren Auseinandersetzungen nicht richtig aufgefasst. Auch sein Ausspruch (ebenda): "Die Annahme, dass die dünnsten Schichlen parallel zur Basis nach verschiedenen Richtungen hin eine verschiedene optische Elasticität besitzen, ist bei wirklich einaxigen, nicht pseudoeinaxigen Krystallen unvereinbar mit der Symmetrie des Systemes " beruht auf einer Verkennung meiner Theorie der Krystallstructur und ist thatstichlich unhaltbar. Wenn Herr Fock endlich die Existenz von Krystallen mit schrauben förmiger Structur für gar nicht wahrscheinlich bält und sich dabei auf die von Herrn Wulff vorgebrachten Gründe beruft, so ignorirt er völlig meine eingehende Widerlegung der W ulff'schen Angriffe (diese Zeitschr. 1888, 14, 417一423). 
Führt man diese Bewegung aus, d. h. dreht man das Ebenenpaar um $120^{\circ}$ um die Axe und verschiebt es zugleich um eine gewisse Strecke längs derselben, und wiederholt man diese Deckbewegung beliebig oft, so werden die Anfangs in's Auge gefassten Punkte in lauter solche Orte geführt, die ebenfalls mit Punkten desselben Systems besetzt sind; und man hat auf diese Art das ganze Punktsystem construirt. Hieraus erkennt man, dass das System in der That ähnlich wie die Glimmercombination von Reusch aufgebaut ist, nur dass die Blättchen um $120^{\circ}$ statt um $60^{\circ}$ gegeneinander gedreht sind. Diese Abweichung ist aber ohne Bedeutung; denn durch Drehung um $120^{\circ}$ um die zum Blättchen senkrechte Axe wird dasselbe in eine Lage gefuhrt, in der es sich gegenüber einem senkrecht zur Blattfläche einfallenden Parallelstrablenbündel genau so verhält, als sei es in entgegengesetztem Sinne um $60^{\circ}$ gedreht worden.

Um dies zu ubbersehen, erinnere man sich, dass man die Fortpflanzungsgeschwindigkeit einer Wellenebene und die Schwingungsrichtung der Theilchen in ihr erhält, wenn man einen zur Wellenebene parallelen Schnitt durch das Centrum der für die gegebene Krystallplatte charakteristischen optischen Elasticitätsfläche hindurchlegt. Dieser Schnitt ist ein Oval, welches zu zwei aufeinander senklechten Durchmessern symmetrisch ist. Die letzteren stellen durch ihre Grösse und Richtung obige beiden Bestimmungsstücke für jede der beiden parallelen, im Krystall fortschreitenden Wellenebenen dar. Bei Umdrehung der Krystallplatte um $180^{\circ} \mathrm{um}$ die zu ibr senkrechte Gerade kommt dieser Ovalschnitt mit sich selbst zur Deckung, so dass optisch kein Unterschied gegen vorher entsteht. - Weil übrigens beim Glimmer die erste Mittellinie der optischen Axen sehr nahe senkrecht zur Blättchenfläche steht, so werden auch in convergentem Lichte die Erscheinungen, welche dasselbe Blättchen in zwei um $180^{\circ}$ gedrehten Lagen darbietet, sehr nahe ubereinstimmen. Daher wird überhaupt in allen Beziehungen das optische Verhalten einer Glimmersäule von $120^{\circ}$ kaum merklich unterschieden sein von demjenigen einer mit $60^{\circ}$, a b e r in entgegengesetztem Drehungssinne, aufgebauten Glimmercombination. - Die r ech t s drehende Glimmercombination von R e u s ch kann man also entweder so schildern, wie oben geschehen, oder statt dessen auch so: Jedes weiter aufgelegte Glimmerblättchen sei gegen das vorhergehende um $120^{\circ}$ i m Uhrzeigersinne gedreht. Die gedachten Punktsysteme sind also geeignet, die Structur rhomboëdrischer drehender Krystalle darzustellen.

Bei dem Versuche, das optische Drehvermögen von Krystallen, die dem quadratis ch en Krystallsysteme angehören, in entsprechender Weise zu deuten, stösst man nun auf eine Schwierigkeit. Auch hier giebt es freilich Punktsysteme, die aus monoklinen Blättchen aufgebaut erscheinen. Aber da diese Systeme vierzählige Schraubenaxen besitzen, so muss man Drehungen um $90^{\circ} \mathrm{um}$ die Hauptaxe, nebst gleichzeitiger Schiebung längs 
dieser Axe, ausführen, um die aufeinander folgenden Blättchen zur Deckung zu bringen. Weil nun ein monoklines Blättchen, welches aus gegebener Anfangslage ein Mal nach rechts herum, das andere Mal nach links herum in seiner Ebene um $90^{\circ}$ gedreht wird, in zwei Lagen gelangt, die sich gegenüber einem senkrecht zur Blattfläche einfallenden Strahle - in nichts von einander unterscheiden, so scheint ein solches Punktsystem denselben Bau wie eine Nörr e m b e rg'sche Glimmersäule mit rechtwinklig gekreuzten Blättchen zu besitzen, und daher unvermögend, die Polarisationsebene eines senkrecht einfallenden Strahles in dem einen oder anderen Sinne zu drehen.

Ein Analogon zu jenen Glimmercombinationen von Reusch, bei denen die Blättchen unter $45^{\circ}$ gegeneinander gedreht sind, scheint man unter den regelmässigen unendlichen Punktsystemen auch nicht suchen zu dürfen, weil achtzähli ge Drehungen oder Schraubungen, d. h. solche mit dem Drehwinkel $\frac{2 \pi}{8}=45^{\circ}$, bei diesen Systemen als charakteristische Deckbewegungen uberhaupt nicht vorkommen.

Auch für die dem regulären Krystallsysteme ange hörigen drehenden Krystalle gelingt es auf dem angedeuteten Wege nicht, die optische Drehung aus der Structur abzuleiten.

Der Grund für diesen Misserfolg liegt in einer unzulässigen Voraussetzung, die bei der oben angestellten Betrachtung gemacht wurde. Bei Vermeidung derselben verschwinden die Schwierigkeiten, wie jetzt für die einzelnen Krystallsysteme nachgewiesen werden soll.

3.

\section{Rhomboëdrisches Krystallsystem.}

1) Das rechte abwechselnde Dreipunktschraubensystem*). (Fig. 1 Taf, VII.) In Fig. 1 (sowie in den folgenden Figuren) stellen die Kreise mit ihren Mittelpunkten die untereinander congruenten Krystallbausteine mit ihren Schwerpunkten vor. Je ein durch einen anderen theilweise verdeckter Kreis bedeutet einen etwas tiefer stehenden Baustein. Die verschieden tiefe Schattirung solcher Kreispaare zeigt verschieden tiefe Lage der Bausteinpaare unter der Zeichnungsebene an, während durch Kreispaare von gleicher Schattirung Baustein paare desselben Abstandes von der Zeichnungsebene angedeutet sind. Die Figur stellt die Projection von sechs aufeinander folgenden parallelen Molekularebenen des Systems auf eine derselben dar, wobei unter "Molekularebene " eine mit Schwerpunkten der Bausleine besetzte Ebene verstanden ist. Diese Schwerpunkte sind in allen

*) Vergl. „Entwickelung “ S. 133, Nr. III B $\beta, 23$. 
Molekularebenen in derselben Weise angeordnet, nämlich so, dass sie ein Netz gleichseitig dreieckiger Maschen bilden. Alle dreizähligen Hauptaxen stehen auf diesen Ebenen senkrecht. Eine Art dieser Hauptaxen geht durch die Mitten der halbregelmässigen*) Sechsecke, die durch sechs nächste Kreiscentra bestimmt sind. Schraubung $\frac{A_{2 \pi}}{3}, \frac{\lambda}{3}$ um eine solche Axe, d. h. Drehung des Systems um $120^{\circ} \mathrm{im}$ Uhrzeigersinne, begleitet von einer Senkung um die Strecke $\frac{\lambda}{3}$, führt die Punkte $11^{\prime}$ in die Lage $22^{\prime}$, diese in $33^{\prime}$, diese in Punkte $1_{0} 1_{0}{ }^{\prime}$, welche sich in dieselben Orte projiciren wie $11^{\prime}$, von diesen aber um $-\lambda$ entfernt sind, u. s. f. Die Gesammtheit dieser Punkte bildet eine zusammengesetzterechte Dreipunktschraube. Die Schar der durch die weissen Kreise (wie $11^{\prime}$ der Figur) dargestellten Krystallbausteine, welche zwei benachbarte Molekularebenen besetzen, hat - fü sich allein betrachtet - den Charakter eines monoklinen Krystalls, sie entspricht also einem Glimmerblättchen. Diese Schicht muss also doppeltbrechend sein, und zwar optisch zweiaxig. Die dreizählige Schraubung führt alsdann diese Blättchen ineinander uber.

Ebenso berechtigt wie diese Auffassung ist aber folgende andere. Man muss beachten, dass die Figur nur einen beliebigen Specialfall dieses Punktsystems darstellt, während das Verhältniss zweier Nachbarseiten des halbregelmässigen Sechsecks der Projectionsfigur jeden beli ebigen Werth von 0 bis $\infty$ haben kann. Also war es völlig willkürlich, dass vorher gerade das durch die Punkte 11' senkrecht zur Hauptaxe gelegte Molekularebenenpaar als ein Krystallblättchen aufgefasst wurde. Mit genau demselben Rechte darf man die durch die beiden Punkte 1'2 senkrecht zur Hauptaxe gelegten Molekularebenen als ein Krystallblättchen betrachten. Dasselbe hat ebenfalls monoklinen Charakter, ist aber von dem vorher betrachteten Blättchen im Allgemeinen verschieden, wenngleich völlige Übereinstimmung nicht ausgeschlossen ist. Natürlich ist es ebenfalls doppeltbrechend, und zwar optisch zweiaxig. Die für das vorliegende Punktsystem charakteristische dreizählige Schraubung führt dies Blättchen in die Lage des durch $2^{\prime} 3$ gelegten Molekularebenenpaares, u.s.f., so dass das ganze System auch als aus di e se n monoklinen Blättchen aufgebaut angesehen werden kann, welche wieder ganz nach Art der Glimmercombination von Reusch, aber wieder unter $120^{\circ}$ gegeneinander gedreht, aufgeschichtet sind.

Da beide Auffassungen des Punktsystems völlig gleichberechtigt sind, so ist die naturgemässe Darstellung desselben folgende: Durch sieben aufeinander folgende Punkte einer unendlichen zusammengesetzten Dreipunkt-

*) „Halbregelmässig « heisst ein Vieleck mit lauter gleichen Winkeln; aber nur abwechselnd gleichen Seiten. 
schraube $14^{\prime} 22^{\prime} 33^{\prime} 4_{0}$ lege man senkrecht zur Hauptaxe Ebenen, so theilen diese den Raum und somit das den Raum erfüllende Medium, den Lichtäther, in sechs Schichten; die erste, dritte, fünfte derselben sind einander gleich, aber um je $120^{\circ}$ gegen einander gedreht; sie haben monoklinen Charakter und sind daher doppeltbrechend optisch zweiaxig. Genau dasselbe gilt von der zweiten, vierten und sechsten Schicht, welche jedoch den ersteren im Allgemeinen nicht congruent sind. Die Symmetrie ebenen je zweier benachbarter monokliner verschiedenartiger Schichten bilden mit einander 600. Das ganze Punktsystem erscheint also wie eine Rechts-Glimmersäule von R usch, indem jedes höhere Blättchen um $60^{\circ}$ entgegen dem Uhrzeigersinn gegen das darunter liegende gedreht ist. Nur darin unterscheidet es sich von dieser Glimmersäule, dass im Allgemeinen nicht sämmtlicheBlättchen, sondern nur je die abwechselnden, einandergleich sind. Im speciellen Falle können jedoch auch alle ubereinstimmen.

Diese Auffassung des Systems ist nicht nur zulässig, sondern nothwendig; denn der Träger der Lichtbewegung ist der jene Schichten erfüllende Aether, der durch die Krystalltheilchen in solchen Zustand versetzt ist, dass je de der aufeinander folgenden Schichten ein monoklines Blättchen und somit doppeltbrechend ist. Eine um die andere dieser Schichten unberücksichtigt zu lassen und für indifferent anzusehen, wie ich es früher gethan, ist unzulässig. Denn während im Glimmerpräparate das Licht beim Verlassen eines Blättchens in den optisch isolropen Copalfirniss und darauf erst in das um $60^{\circ}$ gedrehte folgende Glimmerblättchen eintritt, gelangt es im vorliegenden Punktsystem beim Verlassen einer durch zwei Nachbarmolekularebenen gebildeten doppeltbrechenden Schicht nich $\mathrm{t}$ in eine indifferente, isolrope Schicht, sondern sogleich in die folgende um $60^{\circ}$ gedrebte, ebenfalls dop peltbrechende Schicht, welche freilich der ersteren im Allgemeinen nicht congruent ist.

Dass ein solcher Bau thatsächlich Drehung der Polarisationsebene zur Folge haben muss, wird in $\$ \S 8$ und 9 durch Rechnung und Beobachtung eingehend begründet werden. Hiernach stellt das vorliegende Punktsystem in der That die Structur einer drehenden rhomboëdrischen Substanz dar.

Es giebt auch ein diesem rechten entsprechendes linkes abwechselndes Dreipunktschraubens y ste $m^{*}$ ). Dasselbe unterscheidet sich vom vorigen nur durch den Windungssinn der Schrauben, so dass die Blättchen mit entgegengesetzlem Windungssinne aufgeschichtet sind.

Die Symmetrieverbältnisse dieser beiden Punktsysteme (dreizählige Hauptaxe, drei zweizählige Queraxen, keine Symmetrieebene und kein Symmetriecentrum) stimmen vollständig mit denen der rechten und linken

*) Entwickelung u. s. f., S. 133, Nr. III B $\beta, 24$. 
Quarzkrystalle uberein*). Da ferner in beiden Systemen als besonders häufige Krystallflächen Rhomboëderfächen auftreten müssen***), so sind mit diesen beiden Punktsystemen die Structuren der rechten und linken Quarzkrystalle ermittelt.

2) Das rechte, resp. linke, zusammengesetzte Dreipunktschraubensyste $m^{* * *}$ ) hat sebr ahnlichen Bau wie das vorige. Man braucht in der Fig. 1 nur alle halbregelmässigen Sechsecke um $30^{\circ}$ um ihre Centra zu drehen, so entsteht die Projectionsfigur des vorliegenden Systems. Man sieht unmittelbar, dass auch dieses System als aus zwei abwechselnden Arten von monoklinen Schichten, die senkrecht zur Hauptaxe liegen, aufgebaut angesehen werden kann. Au ch hier bilden die Symme trieebenen je zweier Nachbarschichten $60^{\circ}$ miteinander. Diese Systeme sind also ebenfalls mögliche Structurformen drehender Krystalle des rhomboëdrischen Systems. Für den Quarz kommen sie indessen nicht in Betracht; denn obwohl ihre Symmetrieelemente ebenso wie die der vorigen beiden Systeme mit denen der Quarzkrystalle ubereinstimmen, so musste doch bei ihnen ein aus zwei dreiseitigen Pyramiden gebildetes Dihexaëder vorherrschen; und dies entspricht der Ausbildung des Quarzes nicht.

3) Das rechte, resp. linke, Dreipunktschraubensystem †) unterscheidet sich vom abwechselnden Dreipunktschraubensystem (Fig. 1) dadurch, dass es nicht aus Punkt pa a r e $1 \mathbf{1}^{\prime}$ u. s. f., sondern nur aus einfachen Punkten besteht. Es lässt sich auffassen als aus lauter gleichen $t r i$ k li in e B Bättchen aufgeschichtet, die um $120^{\circ}$ gegeneinander gedreht sind. Beim rechten System ist jedes höhere Blättchen um $120^{\circ}$ gegen den Uhrzeigersinn gegen das darunter liegende gedreht. Dies wurde einer linken Glimmercombination von Reusch ontsprechen. Ueber solche, aus triklinen Blättchen aufgebaute Combinationen liegen allerdings noch keine Beobachtungen vor. Weil die beiden Enden einer Hauptaxe weder deckbar, noch spiegelbildlich gleich sind, so wurde dies System die Structur eines hemimorphen drehenden Krystalles des rhomboëdrischen Krystallsystemes, d. i. einer Form, wie sie das uberjodsaure Natrium besitzt, darstellen.

Ausser den genannten Structurformen finden sich unter den diesem Krystallsysteme entsprechenden Punktsystemen keine anderen, welche schraubenartigen Bau analog der Glimmercombination besässen und daher optisch activ sein könnten.

*) Erweiterung u. s. f. Diese Zeitschr. 1888, 14, 446.

**) Vergl. "Ueber Spaltungsflächen u. s. f." Diese Zeitschr. 1887, 13, 229 ff.

***) Vergl. „Entwickelung “, S. 128, Nr. III B $\alpha, 19$ u. 20.

t) 1. c. S. 96 , Nr. Ill A, 15 u. 16 .. 
4.

\section{Quadratisches Krystallsystem.}

Unter den Punktsystemen mit vierzähliger Hauptaxe sind es zwei, welche als Structuren drehender Krystalle betrachtet werden míssen.

1) Das rechte, resp. linke, zusammengesetzte Vierpunktsch raubens yste m*) (Fig. 2, Taf. VII). Die Figur stellt die Projection von acht auf einander folgenden parallelen Molekularebenen des Systemes auf eine derselben dar. Diese Ebenen sind alle in gleicher Weise mit Schwerpunkten von Bausteinen besetzt, nämlich so, dass letztere ein Netz mit quadratischen Maschen bilden. Alle vierzähligen Hauptaxen stehen auf diesen Ebenen senkrecht. Eine Art der Hauptaxen geht durch die Mitten der halbregelmässigen Achtecke, die durch acht näohste Kreiscentra (der Fig. 2) beslimmt sind. Rechtsschraubung $\left.{ }^{* *}\right) \frac{2 \pi}{4}, \frac{\lambda}{4}$ um eine solche Axe, d. h. Drehung des Systemes im Uhrzeigersinne um $\mathbf{9 0 0}^{\circ}$, gefolgt von einer Senkung um die Strecke $\frac{\lambda}{4}$, führt die Punkte $11^{\prime}$ in $22^{\prime}$, letztere in $33^{\prime}$, diese in $44^{\prime}$, und diese wieder in Orte $1_{0} 1_{0}{ }^{\prime}$, welche um $\lambda$ von $11^{\prime}$ entfernt sind, u. s. f. Die Gesammtheit der genannten Punkte bildet eine rechte zusammengesetzte Vierpunktschraube. Die Schaar der durch die weissen Kreise (wie $11^{\prime}$ der Figur) dargestellten Krystallbausteine, welche zwei benachbarte Molekularebenen besetzen, hat - für sich allein betrachtet - den Charakter eines monoklinen Krystalles. Dasselbe gilt aber auch von der Schaar der durch die Kreise wie 1' 2 dargestellten Bausteine.

Legt man also durch neun auf einander folgende Punkte 1 1' $22^{\prime} 33^{\prime}$ $44^{\prime} I_{0}$ einer zusammengesetzten Vierpunktschraube senkrecht zur Hauptaxe Ebenen, so theilen diese den ganzen Raum in acht Schichten; die erste, dritte, funfte und siebente derselben sind einander $\cong$, aber um $90^{\circ}$ gegen einander gedreht; sie sind monoklin, also optisch zweiaxig. Ganz dasselbe gilt von der zweiten, vierten, sechsten und achten Schicht, welche jedoch den vorigen Schichten nicht congruent sind. Die Symmetrieebenen je zweier benachbarter monokliner verschiedenartiger Schichten bilden miteinander $45^{\circ}$. Das ganze System erscheint also wie eine Rechts-Glimmercombination von $R$ e usc $\mathrm{h}$, bei welcher jedes höhere Blättchen um $45^{\circ}$ gegen den Uhrzeigersinn gegen das darunter liegende gedreht ist. Nurdarin unterscheidet es sich von dieser Glimmersäule, dass nicht sämmtliche Schichten, sondern nurje die abwechselnden, einander $\cong$ sind. Dass auch dieser Bau Drehung zur Folge haben muss, wird eingehend in $\$ \S 8$ und 9 bewiesen.

*) Vergl. "Entwickelung “, S. 118 u. 119, Nr. IV B $\alpha, 32$ u. 33.

**) Die Beschreibung bezieht' sich nur auf das re ch te System. 
Die Symmetrieverhältnisse dieses rechten, resp. linken, Punktsystemes (vierzählige Hauptaxe, vier zweizählige Queraxen, keine Symmetrieebene und kein Symmetriecentrum) stimmen vollständig mit denen der trapezoëdrisch-halbflächigen Abtheilung des quadratischen Syslemes überein, welcher z. B. das optisch active schwefelsaure Strychnin angehört. Somil ist es gelungen, auch die optische Activität von quadratischen Krystallen als nolhwendige Folge ihrer Structur zu begreifen. Es giebt indessen noch eine zweite mögliche Structur drehender quadratischer Krystalle, d. i.

2) Das rechte, resp. linke, abwechselnde Vierpunktschraubensystem*) (Fig. 3). Die Figur stellt die Projection von acht aufeinander folgenden parallelen Molekularebenen des Systems auf eine derselben dar. Alle sind in gleicher Weise mit Schwerpunkten von Bausteinen besetzt, so dass letztere ein Netz mit quadratischen Maschen bilden. Alle vierzähligen Hauptaxen stehen auf diesen Ebenen senkrecht. Eine Hauptaxenart geht durch die Mitten der in der Projectionsfigur auftretenden Quadrate von einer gewissen Stellung, die durch vier nächste Kreiscentra bestimmt sind. Rechtsschraubung***) $A_{\frac{2 \pi}{4}}, \frac{\lambda}{4}$ um eine solche Axe führt die Punkte $11^{\prime}$ in $22^{\prime}$ u.s.f. Die Schaar der durch die weissen Kreise (wie $11^{\prime}$ der Figur) dargestellten Krystallbausteine, die zwei benachbarte Molekularebenen besetzen, hat - für sich allein betrachtet - den Charakter eines monoklinen Krystallblättchens, dessen Symmetrieebene durch die eine Diagonale der durch die Punkte 1 bestimmten Quadrate, sowie durch die vierzählige Hauptaxe des Systems geht. Die Schaar der durch die Kreise wie $1^{\prime}$ und 2 dargestellten Krystallbausteine, die ebenfalls zwei Nachbarmolekularebenen besetzen, hat ebenfalls den Charakter eines monoklinen Blättchens; die Symmetrieebene desselben geht aber durch die in der Figur senkrechte Mittellinie der durch die $1^{\prime}$ bestimmten Quadrate, macht also $45^{\circ}$ mit der Symmetrieebene der vorigen Schicht.

Legt man also durch die neun aufeinander folgenden Punkte der um eine Hauptaxe herumstehenden Punktpaare $11^{\prime} 22^{\prime} 33^{\prime} 44^{\prime} 1_{0}$ senkrecht zur Hauptaxe Ebenen, so theilen diese den ganzen Raum in acht Schichten; die erste, dritte, fünfte und siebente sind einander $\cong$, aber um $90^{\circ}$ gegen einander gedreht; sie sind monoklin, also optisch zweiaxig. Dasselbe gilt von der zweiten, vierten, sechsten und achten Schicht, welche aber den vorigen Schichten nicht $\cong$ sind. Die Symmetrieebenen je zweier benachbarter monokliner verschiedenartiger Schichten bilden miteinander 450. Somit hat auch dieses System völlig den Bau einer rechten, resp. linken, Glimmersäule nach $R$ eusch, jedoch aus Blättchen von

*) Vergl. "Entwickelung “ S. 124 u. 125, Nr. IV B $\beta, 38$ u. 39.

**) Die Beschreibung bezieht sich nur auf das re ch te Syslem. 
abwechselnder Dicke aufgeschichtet. Die Symmetrieverhältnisse stimmen mit denen der drehenden quadratischen Krystalle überein, so dass lelztere also auch die durch dieses System dargestellte Structur besitzen können.

Das rechte, resp. linke, Vierpunktschraubensystem*) dagegen scheint keine mögliche Structur drehender Krystalle zu sein. Es lässt sich nämlich ansehen als gebildet aus lauter $\cong$, unmittelbar aneinander grenzenden triklinen (oder im speciellen Falle monoklinen) Schichten, die unter je $90^{\circ}$ gegen einander gedrebt sind. Ein solcher Bau genügt nach $\S \mathcal{2}$ nicht zur Erzeugung der Drehung.

Von den ubrigen Punktsystemen mit vierzähliger Hauptaxe bietet keines deutlich ausgesprochenen Windungssinn dar, so dass wohl keines derselben als Structur drehender Krystalle in Betracht kommt.

5.

\section{Hexagonales Krystallsystem.}

Optisch active Krystalle mit sechszähliger Hauptaxe sind bisher nicht bekannt. Indessen scheinen gewisse Beobachtungen des Herrn Vernads$\mathrm{k} \mathrm{y}^{* *}$ ) am Trimesinsäuretriäthylester darauf hinzudeuten, dass diese hexagonal-trapezoëdrische Substanz doch mit einem gewissen, wenn auch nicht sehr regelmässigen Drehvermögen begabt ist. Daher hat es ein gewisses Interesse, dass nach meiner Theorie thatsächlich vier verschiedene hexagonale Structuren Drehung zur Folge haben müssen; unter ihnen zwei hemimorphe.

1) Das rechte, resp. linke, zusammengesetzte Sechspunktschraubensystem ${ }^{* * *}$ ) (Fig. 4). Die Figur stellt die Projection von zwölf aufeinander folgenden parallelen Molekularebenen des Systems auf eine von ihnen vor; alle diese Ebenen sind identisch besetzt, so dass die Schwerpunkte der Bausteine ein Netz gleichseitig dreieckiger Maschen bilden. Alle sechszähligen Hauptaxen sind senkrecht zu diesen Ebenen. Die uın eine Hauptaxe zunächst herumstehenden Punkte bilden eine zusammengesetzte Sechspunktschraube. Legt man durch 13 aufeinander folgende Punkte 1 1'2 2'... einer solchen Schraube Ebenen senkrecht zur Hauptaxe, so zerlegen sie den ganzen Raum in zwölf Schichten, von denen die erste, dritte, fünfte, siebente, neunte, elfte unter einander gleich sind, die dazwischen liegenden auch einander gleich, aber verschieden von den vorigen. Jede der beiden Schichtenarten hat geometrisch monoklinen Cha-

*) Vergl, "Entwickelung u. s. f.c, S. 89 u. $90, \mathrm{Nr}$. IV A, 26 u. 27.

**) W. Vernadsk y, Ein Beitrag zur Kenntniss des hexagonalen Krystallsystems. Diese Zeitschr. $1889,15,473$; besonders S. 485 u. 486.

***) Vergl. „Entwickelung «, S. 113 u. 114, Nr. VB, 48 u. 49. 
rakter. Die Symmetrieebenen je zweier benachbarter monokliner verschiedenartiger Schichten bilden miteinander 30 . Also gleicht das rechte System einer rechten Glimmercombination, aufgeschichtet aus zweierlei Arten von Glimmerblätchen, die miteinander abwechseln, und die so liegen, dass die Hauptschnitte jezweier benachbarter $30^{\circ}$ miteinander bilden.

In $\S 8$ werde ich zeigen, dass dieser Bau nothwendig Drehung zur Folge hat.

Das Punktsystem hat dieselben Symmetrieeigenschaften wie die trapezoëdrisch-halbflächige Abtheilung des hexagonalen Systems (sechszählige Hauplaxe, sechs zweizählige Queraxen, keine Symmetrieebene, kein Symmetriecentrum). Denselben Symmetriecharakter besitzt auch das folgende System.

2) Das rechte, resp. linke, zweigängige zusammengesetzte Sechspunktschraubensystem*). Die dieses System darstellende Figur entsteht aus der vorigen, wenn man den Kreisen $44^{\prime} 55^{\prime} 66^{\prime}$ dieselbe Schattirung wie resp. $11^{\prime} 22^{\prime} 33^{\prime}$ ertheilt, so dass sie mit diesen in gleicher Entfernung von der Bildfläche liegend zu denken sind. Dann kommen nur sechs aufeinander folgende Schichten in Betracht, die siebente liegt wieder genau senkrecht über der ersten u. s. f. Diese Schichten haben aber jetzt nicht monoklinen, sondern rhombisch-sphenoidischen Charakter. Jedoch sind wieder nur die abwechselnden Schichten einander gleich. Wie beim vorigen Systeme, so erscheint auch hier jede Schicht gegen ihre benachbarte um $30^{\circ}$ gedreht. Das System stellt also ebenfalls eine drehende Struclur dar.

3) Das rechte, resp. linke, Sechspunktschraubensystem **) unterscheidet sich vom zusammengesetzten Sechspunktschraubensystem (Fig. 4) dadurch, dass es nicht aus Punktpaaren 1 l' $^{\prime}$ u. s. f., sondern nur aus einfachen Punkten besteht. Es lässt sich auffassen als aus lauter gleichen triklinen (im speciellen Falle monoklinen) Blättchen aufgeschichtet, die um $60^{\circ}$ gegeneinander gedreht sind. Das rechte System entspricht, - wenn die Schichten monoklin sind - genau ein er Reusch'schen rechtsdrehenden Glimmercombination von $60^{\circ}$. Die Symmetrieeigenschaften dieses Systems stimmen vollständig mit denen uberein, welche die Hemimorphie der trapezoëdrisch-halbflächigen Abtheilung des hexagonalen Systems charakterisiren (sechszählige Hauptaxe, kein weiteres Symmetrieelement). Hierher gehört nach Herrn Vernadsky der Trimesinsäuretriäthylester.

Denselben Symmetriecharakter besitzt das folgende System.

*) „Entwickelung «, S. 114 u. 115, Nr. V B, 50 u. 51 .

**) 1. c. S. $83, \mathrm{Nr}$. VA, 42 u. 43 . 
4) Das rechte, resp. linke, zweigängige Sechspunktschraubensystem*) unterscheidet sich vom zweigängigen zusammengesetzten Systeme dadurch, dass es nicht aus Punktpaaren, sondern nur aus einfachen Punkten besteht. Die wie vorher (3) unter $60^{\circ}$ gedrehten, einander gleichen Schichten sind triklin oder im speciellen Falle rhombischsphenoidisch. Der Bau ist wieder analog der ursprünglichen Reusch'schen Glimmercombination von $60^{\circ}$, jedoch nicht aus monoklinen Blättchen gebildet. Auch dies System ist eine mögliche Structur drehender hexagonaler hemimorpher Krystalle.

6.

\section{Rhombisches, monoklines und triklines Krystallsystem.}

Von den neun Punktsystemen, die dem rhombischen Krystallsysteme entsprechen**), kommen überhaupt nur jene acht in Betracht, welche wenigstens nach einer Richtung zweizählige Schraubenaxen besitzen. Von den senkrecht zu einer solchen Axe liegenden aufeinander folgenden Molekularebenen geht erst die fünfte durch eine zur Axe parallele Deckschiebung des Systems in die erste uber, so dass die Projection von vier aufeinander folgenden Molekularebenen auf eine von ihnen identisch ist mit der Projection des ganzen Systems.

Jedes dieser Systeme ist also ansehbar als gebildet aus je vier aufeinander folgenden doppeltbrechenden Schichten von verschiedener Stellung. Jedoch ist die Stellung der dritten und vierten Schicht von derjenigen der ersten und zweiten nur durch zweizahlige Drehung um die zu ihnen senkrechte Schraubenaxe verschieden. Für ein zu dieser Axe parallel einfallendes Strahlenbundel ist (vgl. § 2) eine solche Verschiedenheit der Stellung ohne Einfluss, so dass diesen Strahlen immer nur zweierlei abwechselnde Schichten entgegentreten. Da nun hierdurch kein Windungssinn nach rechts oder links bestimmt ist, so kann eine solche Structur auch nicht Drehung der Polarisationsebene zur Folge haben.

Ganz ähnliche Ueberlegungen lehren, dass in den dem monoklinen und triklinen Krystallsystem entsprechenden Punktsystemen ${ }^{* * *}$ ) die Bedingungen zur Hervorrufung einer Drehung der Polarisationsebene ebenfalls nicht gegeben sind.

7.

\section{Reguläres Krystallsystem.}

Die bisher bekannten, mit Drehvermögen begabten Krystalle des regulären Systems sind vi e rt elflä chig; sie besitzen ausser drei aufeinander

*) „Entwickelung “, S. 83, Nr. VA, 44 u. 45.

**) 1. c. S. 135-152, Nr. II B $\alpha \beta \gamma \delta, 5-12$ u. 14.

***) 1. c. S. 102 u. $64, \mathrm{Nr}$. II A, 2-4 und I, 1 . 
senkrechten zweizähligen Axen noch vier dreizählige, welche in die durch die vorigen Axen bestimmten Oktanten mitten hineinragen; es fehlt ihnen aber Symmetrieebene und Symmetriecentrum. Punktsysteme mit diesem Symmetriecharakter bietet nur die Abtheilung VI meiner Theorie dar; von ihren fünf Systemen kommen wohl nur folgende zwei in Betracht:

Das reguläre abwechselnde $Z$ weipunktschraubensystem und: Das regulärezusammengesetzte $Z$ weipunktschraubensystem*).

Jedes Punktsystem der Abtheilung VI ist bekanntlich ansebbar**) als zusammengesetzt aus drei congruenten Punktsystemen des rhombischen Krystallsystems II B, die nach drei senkrechten Richtungen durcheinander gesteckt sind, so dass sie durch dreizählige Drehung um eine der dreizähligen Axen ineinander ubergehen. Zum Zwecke des Studiums der genannten beiden $r$ e gulä $r$ en Systeme sind nun zunächst jene $r h o m b i s c h e n$ Theilsysteme, aus denen sie zusammengesetzt sind, genauer zu betrachten. Wie in $\S 6$ erörtert, hat jedes rhombische, mit einer zweizähligen Schraubenax e begabte Punktsystem (II B) die Eigenschaft, dass von den senkrecht zu dieser Axe liegenden, aufeinander folgenden Molekularebenen immer erst die funfte wieder senkrecht uber der ersten liegt und somit durch eine zur Axe parallele Deckschiebung $\lambda$ in die erste ubergeht. Besitzt das rhombische Punktsystem nun nicht nur nach einer Richtung, sondern nach drei aufeinander senkrechten Richtungen zweizahlige Schraubenaxen, - wie es bei jenen Systemen der Fall ist, die den beiden genannten regulären Punktsystemen zu Grunde liegen, dem abwechselnden rechteckigen $Z$ weipunktschraubensystem zweiter Art, und dem rhombischen Gegenschraubensystem - so gilt das Gesagte für jede dieser drei Axen. Also welche seiner Schraubenaxen man auch ins Auge fassen mag: immer lässt sich die Gesammtheit der Punkte eines solchen rhombischen Systems auffassen als auf congruenten Schaaren von je vier Nachbarmolekularebenen angeordnet, die zu der betreffenden Axe senkrecht sind.

Jedes der beiden regulären Punktsysteme (VI 57 und 58) lässt also folgende Auffassung zu.

Eine zweizählige Schraubenaxe $A_{\frac{2 \pi}{2}}, \frac{\lambda}{2}$ werde vertical gestellt; dann liegt die Gesammtheit der Systempunkte auf congruenten Schaaren von je zwölf horizontalen Nachbarebenen, je vier derselben angehörend einem der drei durcheinander gesteckten Theilsysteme. Erst die dreizehnte parallele Molekularebene liegt wieder senkrecht uber der ersten, durch die Deckschiebung $\lambda$ in sie überfubrbar. Diese 13 Molekularebenen zerlegen den Raum in zwölf Nachbarschichten, deren Beschaffenheit jetzt untersucht wird.

*) „Entwickelung “, S. 158 u. 159, Nr. VI, 58 u. 57.

**i l. c. S. 153 u. 154 . 
Die drei Theilsysteme sind so durcheinander gesteckt, dass die Axen von drei nächsten gleichen Zweipunktschrauben $\frac{A_{2 \pi}}{2}, \frac{\lambda}{2}$ ohne Schnilt zwischen einander hindurchlaufen, gelegen wie drei nicht zusammenhängende, nach drei senkrechten Richtungen verlaufende Kanten eines Würfels von der Kantenlänge $\frac{\lambda}{4}$. Diese Axen mögen als $A^{\prime} A^{\prime \prime} A^{\prime \prime \prime}$ bezeichnet werden; erstere beiden seien horizontal, $\boldsymbol{A}^{\prime \prime \prime}$ vertical.

Nun fassen wir aus obigen zwölf Nachbarschichten eine solche $(a)$ in's Auge, innerhalb deren eine horizontale zweizählige Schraubenaxe, z. B. $A^{\prime}$, verläuft; diese Schicht hat den Charakter eines monoklinen (resp. rhombischen) Krystallblättchens. Die diesseits und jenseits an ihr anliegenden, im Allgemeinen triklinen Schichten $\left(b\right.$ und $\left.b^{\prime}\right)$ mussen durch Ausführung der zweizähligen Schraubung $A^{\prime} \frac{2 \pi}{2}, \frac{\lambda}{2}$ ineinander übergehen, also $\cong$ sein; denn letztere Bewegung ist ja eine Deckbewegung des Systems. Durch dieselbe Bewegung kommt die mittlere Schicht $(a)$ mit sich selbst zur Deckung. Die drei aufeinander folgenden Schichten $b \quad a b^{\prime}$ bilden eine Blättchentriade I, welche durch nebenstehende Fig. 5 veranschaulicht werden mag. Diese Figur ist so zu verstehen: Aus den unendlich grossen Blättchen sind gleich grosse Rechtecke so ausgeschnitten, dass ihre Seiten parallel sind den Schwingungsrichtungen, die für einen senkrecht auf das Blättchen fallenden Strahl bei seinem Eindringen massgebend sind. Bei $b$ und $b^{\prime}$ sind diese Schwingungsrichtungen willkürlich angenommen. - Ganz in derselben Weise muss die andere horizontale Axe $A^{\prime \prime} \frac{2 \pi}{2}, \frac{\lambda}{2}$ inmitten einer analog gebauten, wenn auch im Allgemeinen aus anderen Blättchen $d c d^{\prime}$ unter anderen Winkeln zusammengesetzten Triade II verlaufen (Fig. 6), in welcher $c$ monoklin (resp. rhombisch), $d \cong d^{\prime}$ im Allgemeinen triklin. - Die beiden Triaden liegen mit parallelen Blattflächen so ubereinFig. 5.

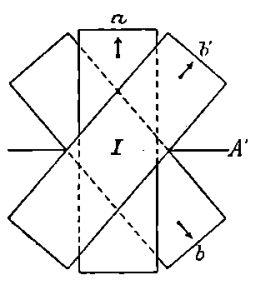

Fig. 6.

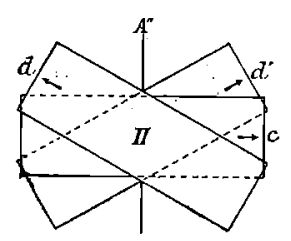
ander, dass die in ihren Mitten verlaufenden Axen $\boldsymbol{A}^{\prime}$ und $A^{\prime \prime}$, um $\frac{\lambda}{4}$ von einander entfernt, sich im Raume rechtwinkelig kreuzen. $A^{\prime \prime}$ sei z. B. die höher gelegene.

Führt man nun die Deckbewegung $A^{\prime \prime \prime} \frac{2 \pi}{2}, \frac{\lambda}{2}$ um die verticale Axe $A^{\prime \prime \prime}$ aus, so werden die Triaden I und II in die neuen Lagen III und IV (Fig. 7 und 8 auf S. 544) gebracht. Hiermit sind die zwölf Molekular- 
schichten construirt, aus deren wiederholter Aufschichtung das ganze System besteht. Dies gilt sowohl für System VI 58 als für VI 5\%. Es sei bemerkt, dass durch Ausführung der Deckbewegung $A^{\prime \prime} \frac{2 \pi}{2}, \frac{\lambda}{2}$, welche die

Fig. 7.

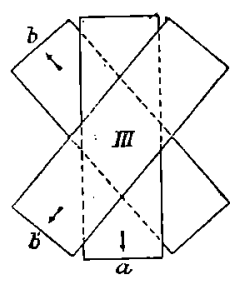

Fig. 8.

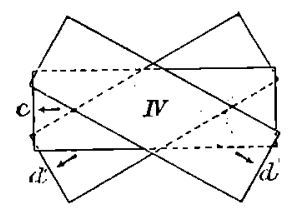

Triade II mit sich selbst zur Deckung bringt, Triade I mit Triade III zur Deckung gelangt, wie leicht zu sehen.

Ein solches reguläres Punktsystem ist also aus vier Arten von parallelen Schichten $a, b, c, d$ zusammengesetzt, von denen $a$ und $c$ monoklin oder rhombisch, $b$ und $d$ im Allgemeinen triklin sind, und welche theils die eine, theils die andere ihrer parallelen Seiten nach oben wenden und dabei noch verschiedene Orientirung besitzen.

Gharakteristisch fur das System ist die Natur der beiden Triaden I und II. Jede derselben zeigt einen bestimmten Drehungssinn der Aufschichtung, welchem offenbar ein bestimmter Sinn der Drehung der Polarisationsebene hindurchgehenden Lichtesentspricht. Jenachdem der Drehungssinn der Aufschichtung beider Triadengleich oder verschieden ist, wirdihre Wirkung auf Hervorrufung optischer Drehung sich addiren oder subtrahiren*).

In $\$ \$ 8$ und 9 wird durch Rechnung und Beobachting bewiesen, dass eine solche Structur Drehung zur Folge haben muss. Doch sei schon hier bemerkt, dass dabei einige Punkte noch unaufgeklärt bleiben.

Welche der in drei aufeinander senkrechten Richtungen vorhandenen zweizähligen Schraubenaxen man auch vertical stellen mag: immer ist das Punktsystem in genau derselben Weise ansebbar als aus congruenten Schaaren von je zwölf zur Verticalaxe senkrechten Schichten aufgebaut, die sich aus denselben Triaden zusammensetzen. Dies folgt aus der Congruenz der Schraubenaxen dieser drei verschiedenen Richtungen. Der Krystall ubt also in jeder dieser drei Richtungen dies elbe Drehwirkung aus. Dass auch fur andere Richtungen Drehwirkung stattfinden muss, ist verständlich, da eine solche ja auch beim Quarz in wenig von der Hauptaxe abweichenden Richtungen vorhanden ist. Hingegen lässt sich nicht unmittelbar einsehen,

*) Gegenüber einem \|| zur Normale einfallenden Parallelstrahlenbündel verbalten sich die Triaden III und IV genau so wie bei I und II, da sie gegen diese nur um $180^{\circ}$ um ibre Normale gedreht sind (vergl. §2). Gegen über einem solchen Parallelstrahlenbündel verhält sich also der Krystall wie eine Blättchencombination, die aus \| aufeinander gelegten abwechselnden Triaden I und II aufgebaut ist. 
dass die Drehung in allen Richtungen de n selben Betrag wie in Richtung der Schraubenaxen haben muss, wie es doch nach den bisherigen Beobachtungen der Fall zu sein scheint.

Es erübrigt noch nachzuweisen, dass, entsprechend einer Structur eines regulären Krystalls, welche $R$ e chts drehung zur Folge hat, jeder Zeit auch eine lin k s dr ehe $\mathrm{n}$ de Structur existirt. - In jedem der beiden behandelten Punktsysteme des regulären Krystallsystems (VI. 58 und VI. 57) laufen zweizählige Schraubenaxen $\frac{A^{2 \pi}}{2}, \frac{\lambda}{2}$ nach drei senkrechten Richtungen ohne Schnitt zwischen einander hindurch, gelegen wie drei nicht zusammenhängende, aufeinander senkrechte Kanten eines Wurfels von der Kantenlănge $\frac{\lambda}{4}$. Hier sind nun zwei Fälle möglich. Fasst man nämlich den Wurfel mit Kante $\frac{\lambda}{4}$ als Rhomboëder auf, und nimmt diejenige seiner Diagonalen, welche jene drei Kanten nicht trifft, zur Rhomboëderhauptaxe, so können diese Kanten, im Uhrzeigersinne herum verfolgt, entweder die drei absteigenden oder die drei aufsteigenden Randkanten sein. - Liegen nun z. B. in einem gegebenen regulären Punktsysteme die Schraubenaxen zu einander wie die absteigenden Randkanten eines Würfels, so erhält man das entsprechende System, in welchem die Schraubenaxen wie die a u f ste i g e n d en Randkanten desselben Würfels verlaufen, einfach dadurch, dass man das Spiegelbild des gegebenen Systems z. B. in Bezug auf eine Spiegelebene, die zweien jener drei Schraubenaxen parallel ist, construirt. Sämmtliche zur dritten Axe senkrechten Schichten werden dabei den entsprechenden Schichten des gegebenen Systems spiegelbildlich gleich. Die Glimmerblättchensảule, welche das neue System nachahmt, ist also der vorigen ebenfalls spiegelbildlich gleich, d. h. der Windungssinn der Aufschichtung ist der entgegengesetzte wie dort. Daher ist dann auch die Drehung der Polarisationsebene zwar gleich stark, aber entgegengesetzt wie dort. Jedes der beiden Punktsysteme VI. 58 und VI. 57 enthält also zwei enantiomorphe Varietäten. (Dies habe ich in meiner „Entwickelung einer Theorie u. s. f. ( nicht ausdrücklich hervorgehoben.)

Soviel uber die Structur der bisher bekannten optisch drehenden Krystalle des regulären Systems!

Unter denjenigen regulären Kryslallen, welche drei aufeinander senkrechte vi e rzäh lige und vier dreizählige Axen besitzen, sind bisher keine oplisch drebenden gefunden. Daber erscheint es vorläufig nicht nothwendig, in erschöpfender Weise zu untersuchen, welche unter den Punktsystemen der Abtheilung VII, die durch ebensolche viel- und dreizählige Symmetrieaxen charakterisirt ist, als Structuren optisch drehender Krystalle 
angesehen werden müssen. Es mag vielmehr genügen darauf hinzuweisen, dass höchst wahrscheinlich das rechte, resp. linke, reguläre Vierpunktschraubensystem*) eine solche Structur darstellt. Dies System ist namlich auffassbar als zusammengesetzt aus drei congruenten abwechselnden Vierpunktschraubensystemen, die nach drei senkrechten Richlungen durcheinander gesteckt sind, so dass drei senkrechte Schraubenaxen $A_{\frac{2 \pi}{4}}, \frac{\lambda}{4}$ wie drei nicht zusammenhängende Kanten eines Würfels von der Kantenlänge $\frac{\lambda}{4}$ verlaufen. Weil nun, wie oben $(\$ 4,2)$ nachgewiesen, schon das einzelne Theilsystem die Polarisationsebene dreht, so ist es mindestens wahrscheinlich, dass auch das aus drei solchen quadratischen Systemen zusammengesetzte reguläre System die Structur eines optisch drehenden Krystalls darstellt.

Ob vielleicht noch andere Systeme dieser Abtheilung VII geeignet sind, die Polarisationsebene zu drehen (etwa Nr. 62, 63, 64, die ja mit vierzähligen gleichen Schraubenaxen begabt sind, welche ohne Schnitt zwischen einander hindurchlaufen, gelegen wie drei senkrechte nicht zusammenhängende Kanten eines Würfels), könnte nur eine eingehendere Untersuchung lehren, zu der aber - wie gesagt — vorläufig durch die Erfahrung kein Anlass gegeben ist.

8.

Alle im Vorigen fur die optisch drehenden Krystalle der verschiedenen Systeme aufgestellten Structurformen haben das gemeinsam: dass sie angesehen werden können als aufgebaut aus doppeltbrechenden Schichten von verschiedener, aber äusserst geringer Dicke, die in regelmässigem Wechsel und mit bestimmtem Windungssinne aufgeschichtet sind. Es bleibt jetzt noch nachzuweisen, dass diese Structurenthatsächlich Drehung der Polarisationsebene bewirken müssen. Dieser Nachweis wird erstlich theoretisch, sodann durch Beobachtungen gefuhrt. Bei der theoretischen Untersuchung bediene ich mich im Wesentlichen der Mallard'schen Methode, welche vor der fruher von mir angewandten grosse Vorzuige besitzt.

Zum Verständniss des Folgenden erscheint es nothwendig, einige Ergebnisse der Mallard'schen Untersuchung**), z. Th. mit kurzer Andeutung der Beweise, vorauszuschicken. Die grundlegende Aufgabe ist die: den Durchgang eines geradlinig schwingenden Strabls durch eine geringe Anzahl aufeinander geschichteter sehr dúnner Krystallblättchen (ein sogenanntes Packet) zu verfolgen.

*) "Entwickelung «, S. 171, Nr. VII, 65 u. 66.

**) Traité de Cristallographie, 1884, 2, S. 262 ff. 
Zu dem Zwecke betrachtet man zunächst den Durchgang eines elliptisch schwingenden Strahles durch ein einzelnes sehr dünnes Krystallblättchen. Man findet leicht, dass das Axenverhältniss der Schwingungsellipse, sowie die Richtung ihrer grossen Axe dabei nur sebr geringe Aenderungen erleiden, nämlich solche von der Grössenordnung der Blältchendicke. - Wenn also ein ge rad lin ig schwingender Strahl senkrecht auf das erste Blättchen eines Packets fällt, so tritt schliesslich aus dem Packet zwar ein elliptischer Strahl, aber die kleine Axe seiner Schwingungsellipse ist sehr klein gegen die grosse. Daher genügt es für das Folgende, überhaupt nur den Fall zu betrachten, dass e in senkrecht auffallender, sehr gestrecktelliptisch schwingender Strahlein dünnes Krystallblättchendurchdringt. Hierbei bezeichne :

$\lambda$ die Wellenlänge des einfallenden Strables in Luft,

$\varepsilon$ die Dicke des Krystallblättchens,

$\delta$ die Verzögerung, welche der eine der durch Doppelbrechung entstandenen Strahlen gegen den anderen erleiden würde, wenn das Blättchen die Dicke 1 hälte; also

$\varepsilon \delta$ die bei Durchlaufung des Blättchens wirklich eintretende Verzögerung,

$\gamma$ den Winkel, den die grosse Axe der einfallenden Schwingung mit dem einen Hauptschnitt des Blättchens, d. h. mit einer der beiden im Blättchen möglichen Schwingungsrichtungen bildet,

$u$ die kleine Halbaxe der einfallenden elliptischen Schwingung.

Während die grosse Halbaxe nichl merklich verschieden von 1 sei, soll $u$ sehr klein, nämlich von derselben Grössenordnung wie die Blättchendicke sein. Dann bewirkt*) der Durchgang durch das einzelne sehr dünne Krystallblättchen folgende Aenderung $d u$ der Grösse dieser kleinen Halbaxe:

$$
d u=\frac{\pi}{\lambda} \varepsilon \delta \sin 2 \gamma .
$$

Und die grosse Ialbaxe ist nach Durchdringung des Blättchens gegen ihre vorige Richtung gedreht um:

$$
d \omega=\frac{2 \pi}{\lambda} \varepsilon \delta \cdot\left(u+\frac{d u}{2}\right) \cos 2 \gamma .
$$

In beiden Ausdrücken sind erst Grössen dritter OrdFig. 9.

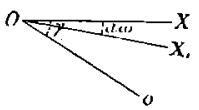
nung vernachlässigt. Mit Rưcksicht darauf, dass $\varepsilon$ und $u$ kleine Grössen erster Ordnung sind, erweist sich die Aenderung $d u$ der kleinen Ellipsenaxe ebenfalls von der ersten Grössenordnung, dagegen die Richtungsänderung $d \omega$ der grossen Axe nur von der zweiten Grössenord-

*) Mallard a, a. O. S. 266 . 
nung. Hiernach darf man, in erster Annäherung, von der durch das einzelne Krystallblättchen bewirkten Drebung absehen.

Jetzt möge ein ursprunglich geradlinig schwingender Strahl ein Packet durchdringen, das aus einer mässigen Anzahl (z) von sehr dunnen Krystallblätchen beliebiger Beschaffenheit aufgeschichtet ist und die Gesammtdicke $d E$ besitzt.

Jedes folgende Blättchen fügt eine neue Aenderung zu der vom vorigen bewirkten Aenderung sowohl der kleinen Ellipsenaxe als der Richtung der grossen hinzu. Die Gesammtänderung, welche beide Grössen beim Austritt des Strahles aus dem Packet aufweisen, heisse $d U$ und $d \Omega$. Es ist also $d U$ der Werth der kleinen Ellipsenaxe des elliptischen Strahles, der aus dem Packet austritt, $d \Omega$ die durch das Packet erzeugte Drehung der grossen Ellipsenaxe. Man sieht unmittelbar, dass die Werthe $d U$ und $d \Omega$ durch Summation der für die aufeinander folgenden Blättchen zu bildenden Einzelwerthe $d u$ und $d \omega$ erhalten werden, also:

$$
\text { 3) } \begin{aligned}
d U & =\frac{\pi}{\lambda} \sum \varepsilon_{p} \delta_{p} \sin 2 \gamma_{p}, \\
d \Omega & =\frac{2 \pi}{\lambda} \sum{ }_{p}\left(u_{p}+\frac{d u_{p}}{2}\right) \varepsilon_{p} \delta_{p} \cos 2 \gamma_{p},
\end{aligned}
$$

wo die $\Sigma$ über alle Blältchen des Packets auszudehnen ist.

Die Grössen $d U$ und $d \Omega$ lassen nun eine einfache geometrische D a rstellung zu, die sich sehr nutzlich erweist. Von einem Punkte $O$ aus ziehe man erstlich senkrecht aufeinander zwei Coordinatenaxen, deren positive Richtungen $O V$ und $O U$ seien, und sodann eine Gerade $\overline{O 1}$, von der Länge $\frac{\varepsilon_{1} \delta_{1}}{d E}$, welche mit $O V$ den Winkel $2 \gamma_{1}$ einschliesst. Im Punkte 1 setze man \begin{tabular}{l} 
Fig. 10. \\
\hdashline
\end{tabular} cissenaxe $O V$ den $\measuredangle 2 \gamma_{p}$ einschliesst. Die Projection der $p$ ersten Linien dieses Linienzuges auf die Ordinatenaxe $O U$ giebt :

$$
\overline{O p^{\prime \prime}}=\frac{\varepsilon_{1} \delta_{1}}{d E} \sin 2 \gamma_{1}+\frac{\varepsilon_{2} \delta_{2}}{d E} \sin 2 \gamma_{2}+\cdots+\frac{\varepsilon_{p} \delta_{p}}{d E} \sin 2 \gamma_{p}
$$

oder mit Rücksicht auf Gleichung 1) 


$$
\overline{o p^{\prime \prime}}=\frac{\lambda}{\pi} \cdot \frac{d u_{1}+d u_{2}+\cdots+d u_{p}}{d E} .
$$

Weil aber $u_{p-1}+d u_{p-1}=u_{p}$ ist, so ist

$$
\overline{O p^{\prime \prime}}=\frac{\lambda}{\pi} \cdot \frac{\left(u_{p}+d u_{p}\right)}{d E} \text {. }
$$

Die Projection des für das ganze Packet gebildeten Linienzuges $O 42 \ldots p \ldots z$ auf die Ordinatenaxe $O U$ giebt also mit Rucksicht auf Gleichung 3)

$$
\bar{o} \overline{z^{\prime \prime}}=\frac{\lambda}{\pi} \frac{d U}{d \bar{E}} .
$$

Ferner ermittle man den Inhalt der Fläche, die zwischen der pten Linie des Linienzuges, ibren beiden Endordinaten und ihrer Projection auf die Abscissenaxe $O V$ eingeschlossen ist. Das arithmetische Miltel der beiden Endordinaten ist nach Gleichung 5)

$$
=\frac{\lambda}{\pi \cdot d E} \cdot \frac{u_{p-1}+d u_{p-1}+u_{p}+d u_{p}}{2}=\frac{\lambda}{\pi d E}\left(u_{p}+\frac{d u_{p}}{2}\right) .
$$

Folglich ist jener Flächeninhalt

$$
s_{p}=\frac{\lambda}{\pi \cdot d E}\left(u_{p}+\frac{d u_{p}}{2}\right) \cdot \frac{\varepsilon_{p} \delta_{p}}{d E} \cos 2 \gamma_{p} .
$$

Trifft man bei Durchlaufung des Linienzuges von $O$ an auf eine Linie, deren Projection auf die Axe $O V$ dem positiven Sinne dieser Axe entgegen durchlaufen wird, so ist für diese der Winkel $2 \gamma_{p}$ absolut genommen $>90^{\circ}$, so dass $\cos 2 \gamma_{p}$ negativ wird. Weil aber $\frac{\lambda}{\pi . d E}\left(u_{p}+\frac{d u_{p}}{2}\right)$ das arithmetische Mittel der beiden Endordinaten bedeutet, so ist dasselbe positiv, solange die fragliche Linie in dem Quadranten der beiden positiven Halbaxen liegt. Folglich ist die Fläche $s_{p}$ in einem solchen Falle negativ zu rechnen.

Die Gesammtfläche, welche zwischen dem ganzen Linienzuge, seiner Endordinate $z z^{\prime}$ und der Abscissenaxe $O V$ enthalten ist, wird mit Rü cksicht auf Gleichung 4)

$$
\text { II) } s=\frac{\lambda}{\pi \cdot d E^{2}} \sum\left(u+\frac{d u}{2}\right) \varepsilon \delta \cos 2 \gamma=\frac{\lambda^{2}}{2 \pi^{2}} \frac{d \Omega}{d E^{2}} \text {. }
$$

Vermöge der Gleichungen I) und II) erscheint die kl eine Ellipsenaxe $d U$ des austretenden Strahles proportional der Projection des für das Packet gezeichneten Linienzuges auf die $U$-Axe; und die erzeugte D reh ung der grossen Ellipsenaxe $d \Omega$ proportional der Fläche, die durch jenen Linienzug, seine Endordinate und die Abseissenaxe $O V$ begrenzt ist. 
Für uns kommen, wie sich zeigen wird, im Wesentlichen nur solche lackele in Frage, für welcho der gébrochene Linienzug 012 . . z ein geschlossenes Vieleck bildet, sodass $z$ in $O$ fält; dieser Fall wird daher hinfort allein untersucht. Unter dieser Voraussetzung ist $O z^{\prime \prime}=0$, also

III)

$$
d U=0,
$$

d. h. die kleine Ellipsenaxe der aus dem Packet austretenden Schwingung ist $=0$ : die Schwingung ist geradliniggeblieben!

Ferner erkennt man, mil Beachtung des uber das Vorzeichen der zu summirenden Flächenstủcke Gesagten, dass für diesen Fall die in Gleichung II) auftretende Fläche $s$ den Inhalt des geschlossenen Vielecks darstellt; er heisse $s_{0}$. Dann ist also:

$$
d \Omega=\frac{2 \pi^{2}}{\lambda^{2}} s_{0} \cdot d E^{2}
$$

Die Drehung der Schwingungsrichtung ist also dem Flächeninhalte des geschlossenen Vielecks proportional. Nun ist die Länge jeder Vielecksseite $=\frac{\varepsilon \delta}{d E}$; aber $\delta$ ist für die verschiedenen Farben nur wenig verschieden. Sieht man es in erster Näherung als unabbängig von der Farbe an, so ist der Flächeninhalt unabhängig von $\lambda$; und die Drehung erscheint in erster Näherung umgekehrt proportional mit $\lambda^{2}$, was der Erfahrung entspricht.

Behufs weiterer Schlüsse ist das Vieleck noch genauer zu untersuchen. Die Richtungen je zweier Nachbarseiten desselben bilden miteinander den $\Varangle 2\left(\gamma_{p}-\gamma_{p+1}\right)$. Hier bedeutet $\gamma_{p}$ den Winkel, den die grosse Axe der auf das $p$ te Blättchen einfallenden elliptischen Schwingung mit dem e in en Hauptschnitt (vgl. S. 547) dieses pten Blättchens einschliesst. Würde die grosse Axe der auf das folgende Blättchen fallenden Schwingung noch unverändert dieselbe Richtung wie beim Einfall auf das vorige Blättchen beibehalten haben, so wäre $\gamma_{p}-\gamma_{p+1}$ nichts anderes als der Winkel, den die entsprechenden Hauptschnitte der beiden aufeinander folgenden Blättchen miteinander einschliessen. Nun ändert sich freilich die Richtung der grossen Axe der Schwingungsellipse beim Durchlaufen des sehr dünnen Krystallblättchens ; aber diese Richtungsänderung ist, wie erwähnt (Gl. 2) nur von der zweiten Grössenordnung, so dass man sie in erster Näherung vernachlässigen kann.

Mit dieser Annäherung darf man also behaupten, dass der Richtungsunterschied zweier Nachbarseiten des Vielecks $O 12 \ldots z$ das Doppelte des Winkels darstellt, den die entsprechenden Hauptschnitte zweier aufeinander folgender Blättchen einschliessen.

Demnach ist der Inhalt des Vielecks nur abhängig von der Beschaffen- 
heit des Packets, d. h. von der Qualität und Anordnung seiner Krystallblättchen, nicht abhängig von der Schwingungsrichtung des einfallenden Strahles. Daher lehrt Gleichung IV), dass die durch das Packet erzeugte Drehung unabhänig ist von der Schwingungsrichtungdes einfallenden Strahles.

Wenn man aus beliebigen sehr dünnen Krystallblättchen irgend ein Packet herstellt, und sodann dieselben Blättchen in derselben Reihenfolge und nur entgegengesetzt gegeneinander gedreht wie zuvor wieder aufschichtet, so wird das neue Packet dem vorigen spiegelbildlich gleich. Dann ist auch das Vieleck, welches die jetzige Drehung bestimmt, dem vorigen spiegelbildlich gleich. War bei Durchlaufung des ersteren im Sinne 012 . . z der Flächeninhalt positiv, so ist bei Durchlaufung des letzteren im Sinne $01 \boldsymbol{z}_{0} \ldots z_{0}$ der Flächeninhalt negativ. Da nun die Drehung $d \Omega$ dem Flächeninhalte proportional ist, so ist sie das zweite Malgleich, aber entgegengesetzt der vorigen Drehung. Positiver Flächeninhalt, welcher bei Aufschichtung entgegen dem Uhrzeiger entsteht, entspricht positiver Drehung, d. h. einer solchen im Uhrzeigersinne.

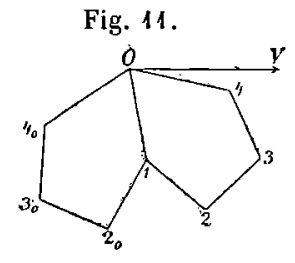

Jetzt sei gegeben eine Säule, die durch Aufschichtung von sehr viele n $(n)$, untereinander identischen, parallel gestellten Packeten hergestellt sei. Ueber die Beschaffenheit des einzelnen Packets wird nur vorausgesetzt,

1) dass seine Krystallblättchen, wenn auch untereinander beliebig verschieden, doch alle se h r dün $\mathrm{n}$ seien, und

2) dass der Endpunkt des für die Drehung massgebenden Linienzuges $012 \ldots z$ mit dem Anfangspunkte zusammenfalle, das Vieleck also geschlossen sei.

Weil unter diesen Umständen das einzelne Packet einen Strahl, der geradlinig schwingend eintritt, auch wieder geradlinig schwingend austreten lässt (Gl. III), so gilt dasselbe von der ganzen Säule: Ein g eradlinig polarisirter Strabl, der in die Säule eintritt, verlasst sie auch geradlinig polarisirt.

Da ferner die Drehung, welche der Schwingungsrichtung des geradlinig schwingenden Strahles durch das einzelne Packet ertheilt wird, unabhängig ist von der anfänglichen Schwingungsrichtung, und, bei gegebenem $\lambda$, nur abhängig von der Beschaffenheit des Packets (Gleichung IV), so ist die nach Durchlaufung von $n$ gleichen Packeten erlangte Drehung $(\Omega)$ $n$ mal so gross als nach Durchlaufung eines einzelnen, also :

$$
\Omega=n \frac{2 \pi^{2}}{\lambda^{2}} s_{0} d E^{2}
$$


oder V)

$$
\Omega=\frac{2 \pi^{2}}{\lambda^{2}} s_{0} \cdot d E \cdot E,
$$

wenn $E$ die Dicke der ganzen Säule. Eine Säule, welche die obigen beiden Bedingungen erfüllt, dreht also die Polarisationsebene hindurchgehenden Lichtes. Diese Drehung ist proportional der Säulendicke, nahe umgekehrt proportional dem Quadrate der Wellenlänge, endlich abhängig von der Beschaffenheit des einzelnen Packets $\left(s_{0} d E\right)$.

Zusätzlich sei bemerkt, dass das Resultat merklich dasselbe bleibt, wenn eine Säule aus $n$ gleichen Packeten und noch einem Bruchtheile eines solchen besteht. Denn die Aenderung, welche ein paar dünne Krystallblältchen sowohl der Ellipticităt des Strahles als seiner Schwingungsrichtung ertheilen, sind nur sehr klein, nämlich von der Grössenordnung der Blättchendicke.

Soll nun dieses Ergebniss Anwendung finden auf die Structurformen, welche im Vorigen fur die drehenden Krystalle aufgestellt sind, so ist nur nachzuweisen, dass diese Structuren als solche Säulen angesehen werden können, welche die obigen beiden Bedingungen erfüllen. Nun sind alle jene Punktsysteme, wie gezeigt, ansehbar als zusammengesetzt aus doppeltbrechenden Blättchen von ungemein geringer Dicke, welch' letztere im Allgemeinen nicht für alle dieselbe ist. Diese Blättchen bilden Packete, die parallel aufeinander geschichtet sind. Somit ist die erste Bedingung erfullt.

$\mathrm{Zu}$ untersuchen bleibt also nur, ob auch die zweite Bedingung erfullt ist: dass der das einzelne Packet charakterisirende Linienzug $012 \ldots z$ ein geschlossenes Vieleck bildet.

Diese Untersuchung wird jetzt für jedes einzelne als Drehstructur hingestellte Punktsystem durchgeführt.

Dabei ist zu beachten, dass die Länge der $p$ ten Vielecksseite nur von der Beschaffenheit des $p$ ten Blättchens $\left(\varepsilon_{p} \delta_{p}\right)$ und von der Packetdicke $d E$ abhängt; ferner dass der Richtungsunterschied zweier Nachbarseiten des Linienzuges doppelt so gross ist als der Winkel, den die Hauptschnitte zweier aufeinander folgender Blättchen einschliessen. In den folgenden Figuren ist immer vorausgesetzt, die Aufschichtung der Blättchen sei links herum (d. h. entgegen der Uhrzeigerdrehung) erfolgt. Dies entspricht einer rechtsdrehenden Structur (und positivem Flächeninhalte des Vielecks), nur beim Dreipunktschraubensysteme einer linksdrehenden Structur.

\section{Rhomboëdrisches Krystallsystem.}

1) Abwechselndes Dreipunktschraubensystem (Taf. VIl, Fig. 1). Pas Packet besteht aus sechs Blättchen; sie sind von zweierlei Art und wechseln regelmässig mit einander ab; die Hauptschnitte zweier 
benachbarter bilden 600. Das charakterislische Vieleck $O 12 \ldots 6$ ist geschlossen (Fig. 12); sein Flächeninhalt setzt sich aus dem der drei congruenten gleichseitigen Dreiecke zusammen.

2) Zusammengesetztes Dreipunktschraubensystem. Ebenso.

3) Dreipunktschrabensystem. Das Packet besteht aus drei gleichen Blättchen unter 120\%. Das Vieleck 0123 Fig. 12.

Fig. 13. schliesst sich zu einem gleichseitigen Dreiecke (Fig. 13).
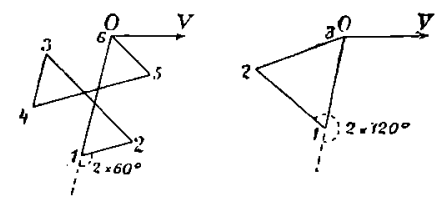

\section{Quadratisches Krystallsystem.}

1) Zusammengesetztes Vierpunktschraubensystem (Taf. VII, Fig. 2). Das Packet besteht aus acht Blättchen; sie sind von zweierlei Art und wechseln regelmässig miteinander $a b$; die Hauptschnitte zweier benachbarler bilden $45^{\circ}$. Das charakteristische Vieleck $012 \ldots 8$ ist geschlossen, nämlich aus zwei sich deckenden Rechtecken gebildet (Fig. 14).

2) Abwechselndes Vierpunktschraubensystem (Taf. VII, Fig. 3). Ebenso.

Fig. 14.

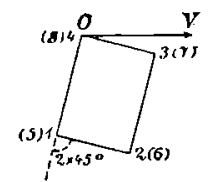

\section{Hexagonales Krystallsystem.}

1) Zusammengesetztes Sechspunktschraubensystem (Taf.VII, Fig. 4). Das Packet besteht aus zwölf Blättchen; sie sind von zweierlei Art und wechseln regelmässig miteinander $a b$; die Hauptschnitte zweier benachbarter bilden $30^{\circ}$. Das charakteristische Vieleck $012 \ldots 12$ (Fig. 15) ist ges chlossen, nämlich aus zwei sich deckenden halbregelmässigen Sechsecken gebildet.

2) Zweigängiges zusammengesetztes Sechspunktschraubensystem. Das Packet besteht aus sechs Blättchen von abwechselnd gleicher Fig. 15. Beschaffenheit, unter dem $\Varangle$ von $30^{\circ}$ gegeneinander gedreht. Das Vieleck $012 \ldots 6$ ist geschloss en; es ist ein halbregelmässiges Sechseck wie Fig. 15.

3) Sechspunktschraubensystem. Das Packet besteht aus sechs gleichen Blättchen, unter $60^{\circ}$ gegeneinander gedreht. Das Vieleck $012 \ldots 6$ ist geschlossen, nämlich aus zwei sich deckenden gleichseitigen Dreiecken gebildet (Fig. 16).

4) Zweigängiges Sechspunktschraubensystem.

Fig. 16.

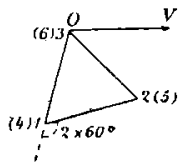


Das Packet besteht aus drei gleichen Blättchen unter $60^{\circ}$. Das Vieleck 0123 ist geschlossen, nämlich ein gleichseitiges Dreieck wie Fig. 16.

\section{Reguläres Krystallsystem.}

1) Reguläres abwechselndes Zweipunktschraubensystem.

2) Reguläres zusammengesetztes Zweipunktschraubens ystem.

Bei beiden Systemen besteht das Packet aus zwölf Blättchen; diese sind von viererlei Art $(a, b, c, d)$ und bilden vier Triaden, die aber paarweise gleich sind. Triade I (Fig. 5, S. 543 ) besteht aus $b, a, b^{\prime}$, die Winkel der Hauptschnitte von $b, a$ und $a, b^{\prime}$ sind gleich $(=\alpha)$. Triade II (Fig. 6) besteht aus $d, c, d^{\prime}$, die Winkel der Hauptschnitte von $d, c$ und von $c, d^{\prime}$ sind gleich $(=\beta)$. $\quad a$ und $c$ sind monoklin, ihre Hauptschnitte bilden miteinander $90^{\circ}$. Auf dieses Triadenpaar folgt ein gleiches, jedoch um $180^{\circ} \mathrm{um}$ die Blättchennormale gegen das vorige gedreht. - Der charakteristische Linienzug $0123 \ldots$ (12) erscheint im Allgemeinen nicht geschlossen;

Fig. 17.

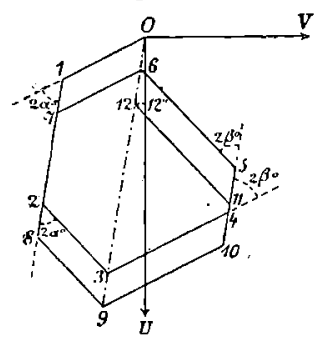
er besteht aus zwei sich theilweise überdeckenden congruenten Theilen (Fig. 17). Die ersten vier Punkte 01 \& 3 bestimmen ein symmetrisches Paralleltrapez; die sich anschliessenden vier Punkte 3456 ein anderes solches. Weil nun die Hauptschnitte der Blättchen $a$ und $c$ aufeinander senkrecht stehen, so müssen die beiden Linien $\overline{12}$ und $\overline{45}$, welche diesen Blättchen entsprechen, $2.90^{\circ}$ mit einander einschliessen, d. h. in entgegengesetzter Richtung verlaufen. So kommen die drei Punkte 0,3 und 6 in eine Gerade. Der folgende Theil des Linienzuges $678 \ldots$ (12) ist dem vorigen deckbar gleich, und die Punkte 9 und 19 fallen auch in die genannte Gerade.

Das Packet übt also jedenfalls Drehung aus; sie ist. nach Gleichung II proportional der Fläche, die durch jenen Linienzug, seine Endordinate und die Abscissenaxe $O V$ begrenzt ist. Dann muss also auch die ganze Säule drehen. Aber weil der Linienzug im Allgemeinen sich nicht völlig schliesst, so erscheint die Drehung nicht völlig unabhängig von der Schwingungsrichtung des einfallenden Strahles; auch muss der das Packet verlassende Strahl s chwach elliptisch sein (Gl. I). Dasselbe gilt dann auch für die ganze Säule. - Aus den bisherigen Beobachtungen an regulären drehenden Krystallen geht nun aber nicht hervor, dass die Schwingungsrichlung des einfallenden Strahles Einfluss auf die Grösse der Drehung hat; auch nicht, dass der austretende Strahl merklich elliptisch ist. Die vorangegangene Betrachtung kann also der Natur nicht völlig entsprechen. In der That: es ist fur die das Packet zusammensetzenden Triaden 
gar keine beschrïkende Annahme gemacht, dieselben sind ganz willkürlich angenommen. In Wahtheit aber geht aus dem Bau jener Punktsysteme hervor, dass die beiden Triaden, ihrer optischen Beschaffenheit nach, gar nicht unabhängig von einander sein können. Da die Art dieser Abhängigkeit aber unbekannt ist, so mussten unsere Annahmen unvollständig bleiben. Es ist durchaus wahrscheinlich, dass man bei vollkommener Einsicht die Triaden von einer solchen Beschaffenheit finden wird, welche vollk ommenen Schluss des das Packet charakterisirenden Linienzuges zur Folge hat.

9.

\section{Versuche.}

Die Versuche auf diesem Gebiete können nie exact sein, sondern höchstens beanspruchen, den in den Krystallen verwirklichten Bedingungen sich einigermassen anzunähern; also kommt ihnen nicht wirkliche Beweiskraft zu. Denn das dünnste Glimmerblättchen, das man bei der Herstellung einer Säule verwendet, ist immer noch sehr dick im Vergleich zu einer der doppeltbrechenden Schichten, aus denen die drehenden Krystalle, der Theorie zufolge, aufgebaut sind. Und die durch Rechnung abgeleiteten Resultate ủber die Drehwirkung von Packeten und Säulen setzen ausdrücklich ungemein dünne Blättchen voraus, und sind um so strenger richtig, je dünner die Blättchen. Die Glimmerpräparate verwirklichen diese Voraussetzung nur in unvollkommener Weise. Daher begreift man, dass manche Glimmercombination noch deutliche Unterschiede in der Ellipticităt des austretenden Strahles, ja auch im absoluten Betrage der Drehung zeigt, je nach der Orientirung (dem Azimuth) der Schwingungsrichtung des einfallenden Strahles gegen das erstgetroffene Blättchen.

Nur mit solchem Vorbehalte dürfen die Versuchsergebnisse als Bestätigung der Theorie herangezogen werden.

\section{Nachahmung rhomboëdrischer drehender Krystalle.}

Sowohl das abwechselade als das zusammengesetzte Dreipunktschraubensystem werden durch eine Glimmercombination nachgeahmt, die aus zwei Arten von Blättchen aufgeschichtet ist, jedes folgende gegen das vorige so gedreht, dass die Hauptschnitte $60^{\circ}$ einschliessen. Aus sogenannten Viertel- und Halbundulationsblattchen, die Hr. Dr. Steeg mir

geliefert hatte, baute ich eine Glimmersäule, in der die 15 dickeren Blättchen regelmässig mit den 15 dünneren wechselten, jedes folgende um $60^{\circ}$ im Uhrzeigersinne gegen das vorige gedreht. Die Beobachtung im parallelen Lichte des Nö r remberg'schen Polarisationsapparates lehrte, dass das Präparat das rothe Licht (eines Rubinglases) um ungefähr $167^{\circ}$ entgegen dem 
Uhrzeigersinne drehte. Die durch solche Drehung des Analysators erzielte Verdunkelung ist sehr vollkommen, sie giebt der beim Quarz kaum etwas nach. Somit ist kaum irgend eine Ellipticität des austretenden Strahles merklich. Durch Drehung des Präparates in seiner Ebene wird die Erscheinung kaum beeinflusst, also hat das Azimuth der einfallenden Schwingung nur ganz geringfügigen Einfluss auf die (überhaupt fast fehlende) Ellipticität des austretenden Strahles. Bei Anwendung weissen Lichtes bewirkt Drehung des Analysators ge gen den Uhrzeigersinn Aenderung der Farbe vom Roth durch alle Spectralfarben bis Violett, zum Beweise, dass das Präparat wirklich links dreht.

Bei Beobachtung im convergenten Lichte war das Azimuth durchaus nicht so einflusslos; denn es trat zwar die für einaxige drehende Krystalle charak teristische Erscheinung auf, aber sie erfuhr sehr merkliche Aenderungen beim Drehen des Präparales in seiner Ebene.

Eine von Herrn Dr. Ste e $g$ in derselben Weise, jedoch aus zwölf Viertel- und zwölf Achtelundulationsblättchen hergestellte Säule zeigte einen wesentlich stärkeren Einfluss des Azimuthes im parallelen Lichte; denn bei manchen Lagen der Säule liess sich nur eine wesentlich unvollkommnere Verdunkelung herbeifuhren. Dafür aber war das Verhalten im convergenten Lichte regelmässiger als das der von mir verfertigten Säule, nämlich gleichwerthig dem Verhalten einer guten Glimmercombination aus lauter gleichen Blättchen.

Das Dreipunktschraubensystem wird durch eine gewöhnliche R e u sch'sche Glimmersäule von $60^{\circ}$ (aus lauter gleichen Blättchen) nachgeahmt; eine solche hat bekanntlich in optischer Beziehung ein sehr quarzähnliches Verhalten, doch zeigt sie im convergenten Lichte noch kleine Aenderungen der Erscheinung bei Drehung des Präparates in seiner Ebene.

\section{Nachahmung quadratischer drehender Krystalle.}

Sowohl das abwechselnde als das zusammengesetzte Vierpunktschra ubensystem werden durch eine Glimmersäule nachgeahmt, die aus zwei Arten von Blättchen aufgeschichtet ist, jedes folgende gegen das vorige so gedreht, dass die Hauptschnitte $45^{0}$ einschliessen. Mir standen zwei solche Präparale zu Gehote, ein selbstgefertigtes und ein von Dr. Steeg geliefertes, in deren jedem 16 Viertelundulationsblättchen mit 16 Achtelundulationsblättchen alterniren, jedes folgende im Uhrzeigersinne gegen das vorige gedreht. Das Steeg'sche Präparat bewirkt Linksdrehung des Roth um elwa 1730; die Verdunkelung bei solcher Drehung des Analysators ist sehr vollkommen und nur unerheblich versehieden bei Drehung des Präparates in seiner Ebene. Das von mir verferligle Präparat war etwas unregelmässig ausgefallen, daher zeigte der austretende Strahl viel grössere 
Ellipticität und stärkere Abhängigkeit vom Azimuth der einfallenden Schwingung. - Im convergenten Lichte sind beide Präparate etwa gleich gut; die beim Drehen des Präparates auftretenden Aenderungen der Erscheinung, die der vom Quarz gebotenen gleicht, sind nicht stark. Auch im weissen Lichte erweisen sich beide Präparate als echte Linksdreher.

\section{Nachahmung hexagonaler drehender Krystalle.}

Sowohl das ein-als das zweigängige zusammengesetzte Sechspunktschra ubens y s tem werden durch eine Glimmersäule nachgeahmt, die aus zwei Arten von Blättchen aufgeschichtet ist, jedes folgende gegen das vorige so gedreht, dass die Hauptschnitte $30^{\circ}$ einschliessen. Ein von Herrn Dr. Steeg bezogenes Präparat, aus 36 Blättchen, nämlich abwechselnd Viertel- und Achtelundulationsblättchen, im Uhrzeigersinne herum (gleich den vorigen) aufgeschichtet, zeigt sehr starke Veränderungen des Drebbetrages und der Ellipticität des austretenden Strahles, wenn es in seiner Ebene gedreht wird. Für verschiedene Azimuthe schwankt die Linksdrehung des Roth von $120^{\circ}$ bis $160^{\circ}$; bei den kleineren Drehungen ist die Verdunkelung ziemlich vollkommen, bei den grösseren aber so gering, dass die Einstellung des Analysators auf maximale Verdunkelung grosse Schwierigkeit bietet. - Im weissen Lichte liefert Drehung des Analysators gegen den Uhrzeiger die furr Linksdreher charakteristische Farbenfolge. Im convergenten Lichte erscheint zwar das Ringsystem mit dem homogenen Mittelfelde, aber statt der vier äusseren schwarzen Kreuzarme sind bei den meisten Azimuthen nur zwei gegentiberliegende angedeutet, bei anderen Azimuthen gar mehr als vier.

Sowohl das ein-als das zweigängige Sechspunktschraubensystem werden durch die ursprünglich von Reusch angegebene Glimmercombination nachgeahmt, die aus gleichen Blättchen unter $60^{\circ}$ aufgeschichtet ist. Dieselbe zeigt bekanntlich die Drehungserscheinungen activer Krystalle recht vollkommen.

\section{Nachahmung regulärer drehender Krystalle.}

Um sowohl das a b w e chselnde als das zusammengesetzte reguläre $Z$ weipunktschraubensystem nachzuahmen, stellte ich die S. 543, Fig. 5 beschriebene Triade I aus zwei Viertel- und einem Halbundulationsblättchen her, deren Axenebenen etwa unter je $40^{\circ}$ gedreht waren; Triade II (ebenda Figur 6) aus zwei Achtel- und einem Viertelundulationsblattchen, etwa unter je $30^{\circ}$ gedreht. In beiden Triaden wurde das nächstfolgende Blättchen i $\mathrm{m}$ Uhrzeigersinne gegen das untere gedreht aufgelegt. Triade II wurde nun auf Triade I in der durch die gegenseitige Stellung der Figuren 6 und 5 dargestellten Weise aufgelegt, so dass die 
optischen Axenebenen der beiden mittelsten Blattchen aufeinander senkrecht waren. Vier solche Doppeltriaden schichtete ich aufeinander, indem ich jede folgende gegen die vorige um $180^{\circ}$ drehte. Das Priparat erwies sich im parallelen Lichte als linksdrebend, wie erwartet, da ja die Aufschichtung - wenn auch ungleichmässig - im Uhrzeigersinne erfolgt war. Bei Drehung des Präparates in seiner Ebene traten starke Aenderungen des Drehbetrages und der Ellipticität des austretenden Strables ein. Die Linksdrehung des Roth variirte für verschiedene Azimuthe von $22^{\circ}$ bis $80^{\circ}$; bei einigen Azimuthen war eine Verdunkelung überhaupt kaum erreichbar, bei anderen war sie sehr vollkommen. Im weissen Lichte ergab Linksdrehung des Analysators die normale Farbenfolge einer linksdrehenden Substanz von Roth durch Grün bis Violett, und zwar bei jedem Azimuthe.

10.

\section{Zusammenfassung.}

In dieser Abhandlung ist bewiesen, dass die von mir entwickelte Theorie der Krystallstructur für die optisch drehenden Krystalle aller Krystallsysteme Structurformen von rechts- und linksdrehendem Charakter darbietet. Abgesehen vom regulären System, ist für alle diese Structurformen der Nachweis geführt, dass der für das betreffende Packet charakteristische Linienzug geschlossen ist, d. h. dass der geradlinig polarisirt einfallende Strahl geradlinig polarisirt bleiben, jedoch eine Drehung seiner Schwingungsrichtung erfahren muss; diese Drehung ist der Dicke der durchsetzten Schicht proportional, dem Quadrat der Wellenlänge nahe umgekehrt proportional. - Für jedes der beiden Punktsysteme des regulären Systems hat sich wenigstens zeigen lassen, dass längs der drei aufeinander senkrechten Hauptaxen Drehung, und zwar von gleichem Betrage, auftreten muss, und dass es je zwei Structuren giebt, welche gleich stark, aber entgegengesetzt drehen.

Die Beobachtungen an Glimmersäulen, welche die drehenden Structuren nachahmen; bestätigen die theoretisch gewonnenen Ergebnisse.

\section{Anhang.}

Die von Herrn Mallard entwickelte Ansicht uber die Structur des Quarz und anderer drehender Krystalle*) ist mit der im Vorstehenden entwickelten nahe verwandt. Obgleich nämlich Herr M a ll a rd zwar im Allgèmeinen an der Bravais'schen Theorie der Raumgitterstructur aller Krystalle

*) Traité de Cristallographie 1884, 2, 313. 
festhält, sieht er sich doch behufs Erklärung der optischen Drehung genöthigt, eine wesentliche Annahme der B rava is'schen Theorie, nämlich die Parallelstellung aller Krystallbausteine, bei den drehenden Krystallen fallen zu lassen*). Diese Annahme allein ist es aber, durch welche man mit Nothwendigkeit zur Raumgitterstructur als der angeblich einzig möglichen Structurform gefuhrt wird. Sobald man diese Annahme fallen lïsst, steht man auf dem Boden der von mir vertretenen Theorie.

Múnchen, im August 1891.

*) A. a. 0. p. 316. „On peut s'étonner que les cristaux de quartz ne puissent se former que par une succession de molecales présentant desorientations régulièrement alternées. On ne connait pas en effet de cristaux de quartz, dans lesquelles les molécules auraient une orientation parallèle etc." Sodann spricht Herr Mallard die Vermuthung aus, Quarzmoleküle in Parallelstellung bildeten den Chalcedon. 